\title{
Percepção dos familiares de pacientes internados em Unidade de Terapia Intensiva em relação à atuação da Fisioterapia e à identificação de suas necessidades
}

\author{
Perception of relatives of patients admitted in Intensive Care Units with regard to the \\ practice of Physical Therapy and identification of their needs \\ Abel Brasileiro de Almeida Neto', Daiana Teresinha Oliveira Evangelista', Fabiana Cristina Tsuda', Marina \\ de Jesus Piccinin', Augusto César Roquejani², Carolina Kosour ${ }^{3}$
}

RESUMO I Trata-se de um estudo descritivo e de abordagem qualitativa, que teve como objetivo verificar o conhecimento dos familiares de pacientes internados na Unidade de Terapia Intensiva do Hospital de Clínicas da Universidade Estadual de Campinas com relação à atuação da Fisioterapia intensiva neste serviço, bem como identificar suas necessidades. Foram entrevistados 60 familiares, sendo estes questionados a respeito do serviço de Fisioterapia e submetidos ao Inventário de Necessidades e Estressores de Familiares em Terapia Intensiva. Foi observado que a maioria deles desconhecia a atuação da Fisioterapia em tal Unidade, porém os que conheciam atribuíram nota máxima ao serviço. Em relação às necessidades dos familiares, notou-se que 56,7\% julgaram necessário saber fatos concretos a respeito do progresso de seu familiar; $60 \%$ gostariam de ter a certeza que seu familiar está recebendo o melhor tratamento e 71,7\% gostariam de ter dias e horários de visitas mais flexíveis. Constatou-se a falta de informações dos entrevistados em relação à atuação da Fisioterapia na Unidade de Terapia Intensiva. Foram identificadas as necessidades dos familiares, sendo possível, com isto, executar ações de melhoria. Por meio do presente estudo, a Unidade de Terapia Intensiva do Hospital de Clínicas da Universidade Estadual de Campinas identificou as principais necessidades dos familiares, adequou o espaço físico, implementou salas de acolhimento familiar e o curso de especialização em Fisioterapia Respiratória em Unidade de Terapia Intensiva Adulto, e criou informativos visuais quanto à sua atuação para melhores acolhimento e compreensão dos familiares.

Descritores I humanização da assistência; Unidade de Terapia Intensiva; Fisioterapia.

\begin{abstract}
I This is a descriptive study, with a qualitative approach, that aimed at verifying the knowledge of relatives of patients hospitalized at the Intensive Care Unit of Hospital de Clínicas at Campinas State University about the performance of the intensive physical therapy, as well as at identifying their needs. We interviewed 60 relatives, questioning them about the Physical Therapy service. The Critical Family Need Inventory was also applied. We observed that most relatives were unaware of the physical therapy team performance at the Intensive Care Unit; however, those who knew about it attributed the highest score to the service. Regarding the relatives' needs, we observed that $56.7 \%$ judged necessary to know the facts about the patients' progress; $60 \%$ would like to be sure that the patient is receiving the best treatment, and $71.7 \%$ would like more flexibility in days and time of visits. It was possible to observe lack of information regarding the action of physical therapy at the Intensive Care Unit and to identify the relatives' needs, allowing improvement actions. The Intensive Care Unit of Hospital de Clínicas at Campinas State University has adapted the physical space and implemented rooms for family reception, with the help of family support and humanization groups. Along with these actions, it was implemented a specialization course in Respiratory Physical Therapy at the Adults Intensive Care Unit and visual aids regarding its importance and actions to a better reception and understanding of the family were distributed
\end{abstract}

Keywords I humanization of assistance; Intensive Care Unit; Physical Therapy specialty.

\footnotetext{
Estudo desenvolvido na Unidade de Terapia Intensiva do Hospital de Clínicas da Universidade Estadual de Campinas - Campinas (SP), Brasil. 'Fisioterapeutas; Especialistas em Fisioterapia em Unidade de Terapia Intensiva (UTI) no Hospital de Clínicas da Universidade Estadual de Campinas (HC-Unicamp) - Campinas (SP), Brasil

2Fisioterapeuta da UTI do HC-Unicamp - Campinas (SP), Brasil.

${ }^{3}$ Fisioterapeuta da UTI do HC-Unicamp; Cocoordenadora do curso de Especialização em Fisioterapia em UTI do HC-Unicamp- Campinas (SP), Brasil. 


\section{INTRODUÇÃO}

As Unidades de Terapia Intensiva (UTIs) foram criadas a partir da necessidade de se concentrarem recursos humanos qualificados e científico-tecnológicos avançados para o atendimento de pessoas criticamente enfermas. Tais serviços passaram a integrar a estrutura hospitalar, com o objetivo de ser proporcionado um ambiente favorável aos profissionais em relação à assistência e à observação contínua dos pacientes internados ${ }^{1-3}$. Porém, as UTIs são caracterizadas como um ambiente 'hostil', em função da intensidade das situações, tais como: o risco de morte, a necessidade de rotina acelerada da equipe de trabalho e a presença de ruídos sonoros ${ }^{4,5}$. Ansiedade e medo são frequentemente encontrados entre os pacientes críticos, tornando relevante a identificação dos estressores que contribuem para o desenvolvimento destes quadros ${ }^{6}$

Gondim et al. definem humanização como um conjunto de medidas que objetivam a conciliação entre os cuidados em saúde e as tecnologias, como também um espaço físico favorável e a satisfação dos profissionais da equipe e dos usuários ${ }^{7}$

A equipe tem papel fundamental no apoio ao familiar, é importante que sejam percebidas as diversas formas como a família pode reagir à nova situação e de que modo a enfrenta. Os sentimentos e os comportamentos da família estão baseados numa série de valores, tais como: a percepção prévia do hospital e da UTI; a gravidade do paciente; a confiança ou não na equipe que cuida de seu ente e que fornece as informações; os aspectos culturais e a maneira que ocorrem as doenças ${ }^{8}$.

A Fisioterapia tem ampla atuação nas UTIs, porém muitos familiares a desconhecem. $\mathrm{O}$ esclarecimento com relação à atuação da Fisioterapia pode ajudá-los, dando mais segurança e conforto.

Também com o intuito de estratificar as necessidades dos familiares de pacientes internados na UTI, a enfermeira Jane Leske, em 1986, criou um questionário com 45 itens de necessidades de acordo com seu grau de importância, a fim de identificar as necessidades percebidas pelos familiares dos pacientes internados em UTI. Esse questionário recebeu o nome de Critical Care Family Needs Inventory (CCFNI). Em 1991, Leske conduziu um estudo com 677 familiares, utilizando o CCFNI e, após análise, os itens foram dispostos em cinco dimensões: suporte, conforto, informação, proximidade e segurança. No Brasil, o CCFNI foi adaptado e validado, denominado de Inventário de Necessidades e Estressores de Familiares de Pacientes Internados em Terapia Intensiva (INEFTI)
Diante da importância do cuidado humanizado e da assistência fisioterapêutica, o presente estudo teve como objetivo verificar o conhecimento dos familiares de $\mathrm{pa}^{-}$ cientes internados na UTI do Hospital de Clínicas da Universidade Estadual de Campinas (HC-Unicamp) com relação à atuação da Fisioterapia intensiva neste serviço, bem como identificar as necessidades dos mesmos, para realizar medidas de melhoria ao acolhimento dos mesmos junto à equipe multiprofissional da instituição.

\section{METODOLOGIA}

O presente estudo foi aprovado pelo Comitê de Ética em Pesquisa da Faculdade de Ciências Médicas da Unicamp (CEP/FCM), sob o parecer no 403/2010. O termo de consentimento livre e esclarecido (TCLE) foi obtido por escrito com os familiares envolvidos na pesquisa.

AUTI do HC-Unicampé composta por 55 leitos, sendo sete de neurologia clínica e cirúrgica, dez de pós-operatório geral, cinco de unidade coronariana, 20 de emergências clínica e cirúrgica, e sete subdivididos em transplante e cirurgia gástrica. Foram selecionadas as unidades de pós-operatório geral, neurocirurgia e cirurgia gástrica.

Trata-se de um estudo descritivo de abordagem qualitativa, no qual foram incluídos 60 familiares de pacientes internados na UTI do HC-Unicamp. Os critérios de inclusão foram: ter um familiar internado na UTI por no mínimo 24 horas; ter visitado o paciente pelo menos uma vez durante o período de internação; idade igual ou superior a 18 anos; ambos os sexos; todas as religiões e crenças e ser responsável direto pelo paciente. Foram excluídos familiares grávidas, pessoas institucionalizadas e com déficit de compreensão, sendo estes indivíduos considerados vulneráveis pelas diretrizes de ética em pesquisa.

Logo após a leitura do TCLE e a concordância dos indivíduos em participarem da pesquisa, foram coletados alguns dados: idade, sexo, tipo de parentesco, grau de escolaridade e tempo de internação do paciente. Para a análise das necessidades dos familiares, aplicou-se um instrumento adaptado do CCFNI, o INEFTI, constituído por 45 itens, organizados em quatro categorias. A primeira é a 'necessidade de conhecimento/informação', itens relacionados às vontades dos familiares em precisarem receber informações em relação ao estado de saúde ou aos procedimentos envolvidos na terapêutica do familiar enfermo. A segunda é a de 'conforto', relacionada ao planejamento físico da unidade, mobília e conforto dos familiares na sala 
de espera da UTI. A terceira é a de 'segurança emocional', direcionada aos sentimentos de segurança, ansiedade e estado de saúde do familiar, e a quarta, 'de acesso ao paciente e aos profissionais da unidade' (médicos, enfermeiros, fisioterapeutas e outros profissionais).

O familiar participante foi abordado na sala de acolhimento familiar da UTI do HC-Unicamp, onde recebeu as informações e os objetivos do presente estudo. Cada participante recebeu o TCLE juntamente com o questionário INEFTI. Foram avaliados também o serviço prestado pela equipe de Fisioterapia do HC-Unicamp, e se os entrevistados tinham conhecimento sobre o serviço prestado, julgando-o como "Bom" ou "Ruim" e atribuindo uma nota por meio da escala pontuada de zero a dez, sendo o valor superior referente ao maior grau de satisfação.

\section{Análise estatística}

Os dados coletados foram submetidos à estatística descritiva e apresentados como forma de números absolutos e percentuais, por meio do programa SPSS, versão 15.0 para Windorws.

\section{RESULTADOS}

\section{Características demográficas dos familiares}

Dos 60 familiares que participaram do estudo, $71,7 \%$ eram do sexo feminino e $28,3 \%$ do masculino, sendo $23,3 \%$ esposas, $21,7 \%$ filhas, $15 \%$ filhos, e os outros $40 \%$ irmãos, mãe, marido, netos e tios. Do total, $45 \%$ dos familiares eram de religião católica, 21,7\% evangélica, e os 33,3\% restantes de religiões espírita, umbanda, ecumênico, caldó ou de nenhuma. Em análise do grau de escolaridade, 38,3\% dos entrevistados tinham concluído o ensino médio; $25 \%$, o superior; e $21,6 \%$, o fundamental; $13,3 \%$ deles tinham ensino fundamental incompleto ou sem escolaridade e 1,76\% não responderam. A média de idade dos participantes foi de 44,3 $\pm 14,63$ anos.

A maioria dos familiares entrevistados (81\%) não possuía convênio médico. Destes, $44(73,33 \%)$ mantiveram-se internados com mediana de $8(5 ; 16,75)$ dias dependentes do serviço da UTI, e 16 (26,66\%) não souberam relatar. Quanto ao atendimento e funcionamento em geral das unidades relacionadas à pesquisa, observou-se que $79 \%$ dos entrevistados atribuíram a este nota dez.

\section{Fisioterapia}

Em relação ao serviço prestado pela equipe de Fisioterapia, somente 35\% dos familiares conheciam o serviço prestado na UTI, e o julgavam como “Bom”. Do total, 20\% atribuíram nota dez ao atendimento fisioterapêutico.

\section{Inventário de necessidades e estressores de familiares em terapia intensiva}

A Tabela 1 mostra a necessidade de conhecimento ou informação dos familiares de pacientes internados em Unidade de Terapia Intensiva, Hospital de Clínicas da Universidade Estadual de Campinas, Campinas, São Paulo, Brasil, com n=60.

Segundo os dados da Tabela 2, estar em um lugar agradável, que ofereça conforto enquanto aguarda para receber notícias de seu familiar, é um fator importante para a maioria dos participantes.

Os participantes se preocupam com seus parentes internados e vivenciam o medo e a insegurança, muitas vezes resultados da incerteza em relação à conduta e ao tratamento, como pode ser observado na Tabela 3.

A Tabela 4 demonstra que $71,7 \%$ dos familiares necessitar dias e horários de visitas mais flexíveis e maior acesso aos profissionais e pacientes, apesar de julgarem que este tempo estabelecido pela instituição supre suas necessidades.

Tabela 1. Necessidade de conhecimento/ ou informação dose familiares de pacientes internados em Unidade de Terapia Intensiva, UTI, Hospital de Clínicas da Universidade Estadual de CampinasC/UNICAMP, Campinas, São Paulo, Brasil (n=60)

$\begin{array}{lcc}\text { Necessidade de conhecimento/informação } & \mathrm{n} & \% \\ \text { Saber fatos concretos sobre o progresso do meu familiar } & 34 & 56,7 \\ \text { Ser informado em casa sobre mudanças da condição do meu familiar } & 33 & 55,0 \\ \text { Conhecer os profissionais que estão cuidando do meu familiar } & 33 & 30 \\ \text { Receber informação sobre o estado de saúde do meu familiar pelo menos uma vez por dia } & 27 \\ \text { Saber o porquê determinado procedimento foi realizado } & 50,0 \\ \text { Saber exatamente o que está sendo feito pelo meu familiar } & 26\end{array}$


Tabela 2. Necessidade do conforto de familiares de pacientes internados em Unidade de Terapia Intensiva, Hospital de Clínicas da Universidade Estadual de Campinas, Campinas, São Paulo, Brasil (n=60)

$\begin{array}{lcc}\text { Necessidade de conforto } & \mathrm{n} & \% \\ \text { Ter um toalete próximo à sala de espera } & 25 & 41,7 \\ \text { Ter um telefone próximo à sala de espera } & 25 & 41,7 \\ \text { Ter a sala de espera próxima do meu familiar } & 22 & 36,7 \\ \text { Ter mobília confortável na sala de espera } & 20 & 33,3 \\ \text { Ter disponível no hospital uma boa comida } & 18 & 90,0 \\ \text { Ter um lugar no hospital onde possa ficar sozinho(a) } & 9\end{array}$

n: número de relatos entre os 60 familiares

Tabela 3. Necessidade de segurança emocional dos familiares de pacientes internados em Unidade de Terapia Intensiva, Hospital de Clínicas da Universidade Estadual de Campinas, Campinas, São Paulo, Brasil $(n=60)$

$\begin{array}{lcc}\text { Necessidade de segurança emocional } & \mathrm{n} & \% \\ \text { Ter certeza que o meu familiar está recebendo o melhor tratamento } & 36 & 60,0 \\ \text { Sentir que há esperança } & 29 & 48,4 \\ \text { Sentir-se acolhido pela equipe multidisciplinar } & 29 & 48,3 \\ \text { Receber a visita de um representante religioso (padre, pastor ou rabino) } & 29 & 24 \\ \text { Conversar sobre a possibilidade de falecimento do meu familiar } & 48,3 \\ \text { Ter alguma outra pessoa comigo quando estiver visitando meu familiar na Unidade de Terapia Intensiva } & 22\end{array}$

n: número de relatos entre os 60 familiares

Tabela 4. Necessidade de maior acesso ao paciente e aos profissionais da unidade de pacientes internados em Unidade de Terapia Intensiva, Hospital de Clínicas, Universidade Estadual de Campinas, Campinas, São Paulo, Brasil ( $n=60)$

$\begin{array}{lcc}\text { Necessidade de maior acesso ao paciente e aos profissionais da unidade } & \mathrm{n} & \% \\ \text { Ter o dia e o horário de visitas flexíveis, podendo ser modificados em situações especiais } & 43 & 71,7 \\ \text { Ter uma pessoa específica no hospital para entrar em contato e ter notícias do meu familiar quando não puder } & 39 & 65,0 \\ \text { estar presente na visita } & 34 & 56,7 \\ \text { Conversar com o médico diariamente } & 30 & 50,0 \\ \text { Visitar meu familiar frequentemente } & 30 & 50,0 \\ \text { Conhecer os cuidados da equipe com relação ao meu familiar } & 25 & 41,7\end{array}$

n: número de relatos entre os 60 familiares

\section{DISCUSSÃO}

Nas UTIs, na maioria das vezes, ocorre o afastamento dos familiares da convivência com seus parentes que se encontram debilitados. As informações são restritas e esta separação é praticamente imposta. Da mesma forma que o ambiente da UTI tende a ser extremamente hostil para o paciente, é mais ainda para os familiares, pois estão aptos a perceber com mais detalhes o ambiente desconhecido e agitado. Costa et al. aplicaram o INEFTI a 53 familiares de pacientes internados em UTI e observaram que o grau de escolaridade do familiar pode interferir nas respostas fornecidas, fenômeno que pode ser explicado devido ao maior acesso às informações dos indivíduos com maior grau de instrução ${ }^{6}$.

Devido ao fato de o $\mathrm{HC}$ ser um hospital público, o mesmo atende em sua maioria pacientes que não possuem convênio médico, sendo estes atendidos pelo Sistema Único de Saúde (SUS), que foi criado no Brasil em 1988, com o objetivo de tornar o acesso à saúde direito dos cidadãos brasileiros. Com a implantação do sistema, o número de beneficiados passou de 30 milhões para 190 milhões de pessoas, sendo que $80 \%$ destes dependem exclusivamente do SUS para ter acesso aos serviços de saúde ${ }^{10,11}$.

\section{Fisioterapia}

Observou-se no presente estudo a falta de conhecimento dos familiares com relação à atuação do profissional fisioterapeuta, que cuida de seus parentes.

No HC-Unicamp, a equipe de Fisioterapia orienta os pacientes e seus familiares no pré e pós-operatório, porém não participa ativamente nos programas de acolhimento familiar. Dos familiares entrevistados, poucos conheciam o serviço, justificando a necessidade de uma abordagem com esclarecimentos concernente aos atendimentos prestados. No entanto, poucos que conheciam atribuíram nota dez ao serviço da Fisioterapia. Devido à esta necessidade, foi confeccionado um folder autoexplicativo descrevendo a atuação da Fisioterapia na UTI, sua importância, juntamente com seus objetivos e técnicas mais utilizadas (Figura 1). Este material foi aplicado a uma amostra 
Orientar os pacientes e familiares, quanto a fisioterapia.

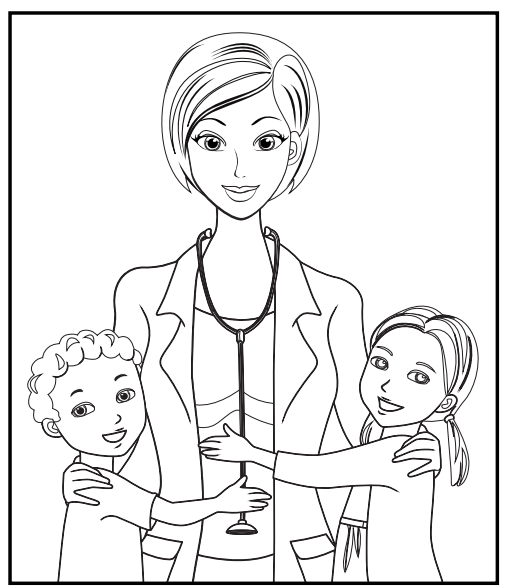

Desenvolver atividades educacionais, como formação de profissionais capacitados para atuar em UTI.

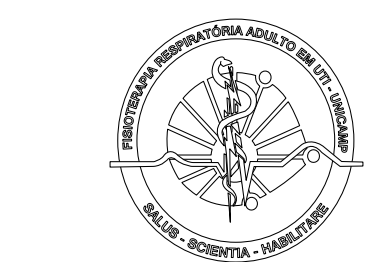

\section{Curso de Especialização de Fisioterapia Respiratória em UTI adulto}

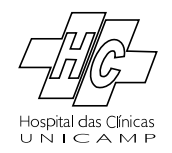

Elaborado por: Carolina Kosour Augusto C. Roquejani Abel B. A. Neto

Daiana T. O. Evangelista

Fabiana C. Tsuda

Marina de Jesus Piccinin

Colaboração:

Equipe Multiprofissional UTI/HC/Unicamp Equipe de Humanização UTI/HC/Unicamp

\section{Como?}

Ajudando na eliminação de catarro, diminuindo a chance de pneumonia. Com manobras de fisioterapia como a vibrocompressão (que ajuda a tirar o catarro).

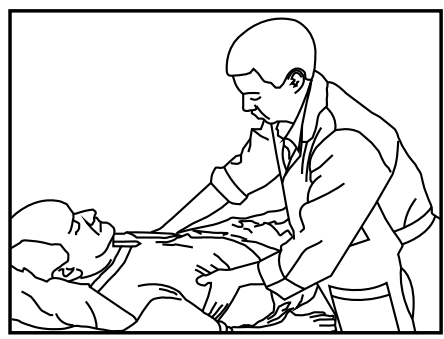

Na melhora da respiração com exercícios, equipamentos e recursos.

A equipe de fisioterapia da UTI faz atendiment ao paciente junto com a equipe de médicos, enfermeiros e funcionários elaborando programas de tratamento e oferecendo assitência fisioterapêutica.

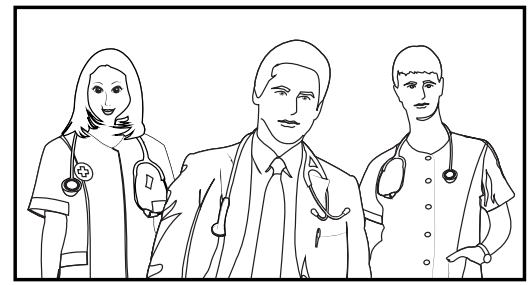

A) Ventilação não-invasiva: é um equipamento que utiliza máscara e é usado para melhorar a respiração e diminuir a falta de ar dos pacientes.

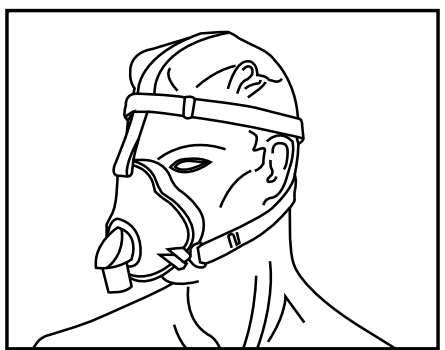

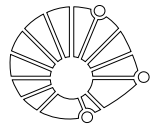

UNICAMIP

$\mathrm{O}$ que a Fisioterapia faz em UTI?
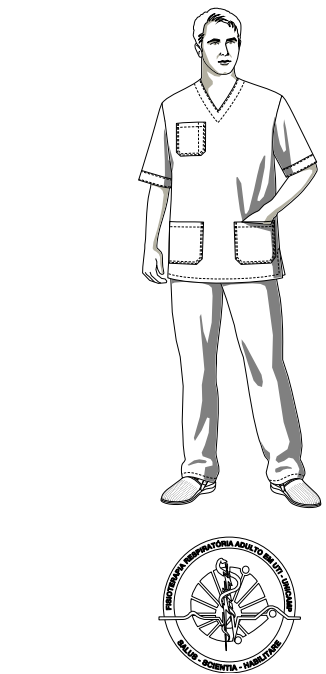

Curso de Especialização de Fisioterapia Respiratória em UTI adulto

2011

B) Inspirometro de Incentivo (Respiron): equipamento com 3 bolinhas, que ajuda na respiração podendo aumentar o folego do paciente.

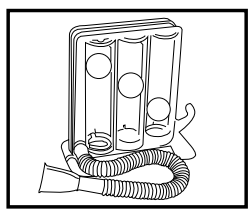

C) Shaker (cachimbo): equipamento usado para ajudar na eliminação de catarro dos pulmões.

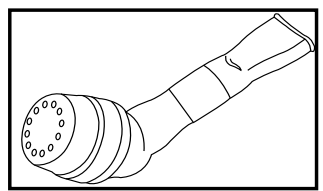

Ajudando o paciente quando estiver precisando de ventilação mecânica, ajustando parâmetros e na retirada da mesma.

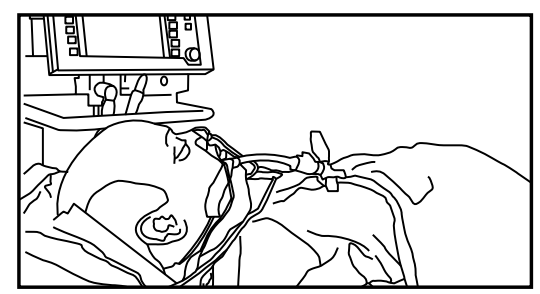

Além da movimentação, posicionamento, conforto, retirada do leito e caminhada. 
de profissionais do HC-Unicamp (médicos, enfermeiros, fisioterapeutas e profissionais da administração) e familiares, sendo considerado 'de fácil compreensão' e 'autoexplicativo' pelos entrevistados.

O fisioterapeuta desempenha papel ativo, juntamente com a equipe multidisciplinar, na implementação de ventilação mecânica invasiva e não invasiva, no processo de desmame ventilatório, no treinamento muscular e na mobilização precoce, dentre outras coisas, visando o suporte clínico adequado ao paciente ${ }^{12-14}$.

$\mathrm{Na}$ maioria dos hospitais em países desenvolvidos, a Fisioterapia é tida como parte integrante da gestão de pacientes nas UTIs. No Brasil, a função exata que os fisioterapeutas exercem varia consideravelmente devido à classificação das UTIs implantadas pelo Ministério da Saúde, classificando-as de acordo com a incorporação de tecnologia, a especialização dos recursos humanos e a área física disponível ${ }^{14,15}$. De acordo com a Resolução RDC no 7 da Agência Nacional de Vigilância Sanitária (Anvisa), institui-se que haja pelo menos um fisioterapeuta para cada dez leitos. Sendo assim, o profissional deve estar disponível em tempo integral para assistência aos pacientes, durante o horário em que estão escalados para atuação na UTI ${ }^{16}$.

\section{Inventário de necessidades e estressores de familiares em terapia intensiva}

No domínio 'necessidade de conhecimento/informação', 56,7\% dos familiares relataram maior necessidade de informações a respeito do progresso do seu familiar, seguido por ser informado em casa sobre mudanças da condição de seu familiar, com 55\%. Esses resultados são similares àqueles encontrados em outro estudo nacional, no qual $84,6 \%$ dos pacientes relataram saber fatos concretos sobre o progresso do paciente como o de maior importância ${ }^{6}$.

Quanto à 'necessidade de conforto', observa-se que das seis necessidades deste domínio, duas foram citadas por mais de $40 \%$ dos familiares, incluindo: 'ter um toalete próximo à sala de espera' e 'ter um telefone próximo à sala de espera'. Tais achados demonstram a importância que os familiares atribuem ao espaço físico da sala de espera de uma UTI, os quais corroboram com o estudo de Maruiti et al. ${ }^{17}$.

No quesito 'necessidade de segurança emocional', ter certeza que o paciente está recebendo o melhor tratamento foi relatado pela maioria dos familiares (60\%). Segundo Costa et al., isto reflete a necessidade do ser humano de sentir-se seguro, uma vez que a dúvida é fonte de sentimentos como a ansiedade e a angústia ${ }^{6}$.

Alguns familiares (35\%) sugerem tempo de visita mais amplo que o habitual, atualmente estipulado em duas horas diárias, porém consideram que o período recente atende às suas necessidades. Estudos nacionais indicam instituições que oferecem apenas uma hora de visita diária, outros serviços dispõem de uma hora e trinta minutos diários ${ }^{18,19}$, demonstrando que o horário de visitas do HC-Unicamp atende à média de outras instituições.

Neste estudo, dentre os dez domínios das 'necessidades de maior acesso ao paciente e aos profissionais', três foram relatados por mais de $50 \%$ dos familiares: disponibilizar dia e horário de visitas flexíveis $(71,7 \%)$, ter uma pessoa específica no hospital para fornecer notícias sobre o paciente (65\%) e conversar com o médico diariamente $(56,7 \%)$. Tais resultados sugerem a importância que os familiares atribuem às informações e ao contato direto com os profissionais que cuidam do seu ente querido ${ }^{20}$.

Em 2010, Costa et al. realizaram um estudo transversal e descritivo com 53 familiares de pacientes internados em UTI. Foi desenvolvida uma escala baseada no conteúdo do Intensive Care Unit Environmental Stressor Scale (ICUESS), na qual os participantes determinavam o grau de estresse de cada item por meio de uma classificação de valores, pontuando de zero a três. Os autores observaram que os itens relacionados ao paciente mostraram maior pontuação por $66,1 \%$ dos entrevistados, seguidos por horário de visitas e contato com a equipe. Concluiu-se que conhecer os eventos estressantes vivenciados pelos familiares pode facilitar as estratégias de humanização hospitalar, propiciando alternativas para reduzir tais níveis de estresse ${ }^{6}$.

\section{CONCLUSÃO}

As principais necessidades dos familiares foram conhecer fatos concretos sobre o progresso do familiar, ter a certeza de que o familiar está recebendo o melhor tratamento, toalete próximo à sala de espera e dia e horário de visitas flexíveis. Houve implementação de salas de acolhimento familiar, com auxílio de grupos de apoio aos familiares e de humanização, além da implementação de informativos visuais. 


\section{REFERÊNCIAS}

1. Domingues $\mathrm{Cl}$, Santini L, Silva VEF. Orientação aos pacientes e familiares: dificuldades ou falta de sistematização. Rev Esc Enferm USP. 1999;33(1):39-48.

2. Pupulim JSL, Sawada NO. Exposição corporal do cliente no atendimento das necessidades básicas em UTI: incidentes críticos relatados por enfermeairas. Rev Latino-am Enfermagem. 2005;13(3):388-96.

3. Pupulim JSL, Sawada NO. Implicações da exposição corporal do cliente na unidade de terapia intensiva durante a visita da família. Cienc Cuid Saúde. 2004;3(1):55-64.

4. Echer IC, Onzi MR, Cruz AMP, Ben GM, Fernandes TS, Bruxel VM. Opinião de visitantes sobre a sistemática de visitação a pacientes internados em unidade de terapia intensiva. Rev Gaúcha Enferm. 1999;20(1):57-68

5. Morgon FH, Guirardello EB. Validação da escala de razão das necessidades de familiares em unidades de terapia intensiva. Rev Latino-am Enfermagem. 2004;12(2):198-203.

6. Costa JB, Felicetti CR, Macedo Costa CRL, Miglioranza DC, Osaku EF, Versa GLGS, et al. Fatores estressantes para familiares de pacientes criticamente enfermos de uma unidade de terapia intensiva. J Bras Psiquiatr. 2010;59(3):182-9.

7. Gondin CT, Souza MFB, Albuquerque NMCV. Cuidado humanizado uma prática possível. Rev Nurs. 2004;71(7):208-11.

8. Martins JJ, Nascimento ERP, Geremias CK, Schneider DG, Schweitzer G, Neto HMO. Acolhimento à família na unidade de terapia intensiva: conhecimento de uma equipe multiprofissional. Rev Eletr Enferm 2008;10(4):1091-101.

9. Freitas KS, Kimura M, Ferreira KASL. Necessidades de familiares de pacientes em unidades de terapia intensiva: análise comparativa entre hospital público e privado. Rev Latino-Am Enferm. 2007:15(1):61-70
10. Brasil. Instituto Brasileiro de Geografia e Estatística - IBGE [Internet]. Pesquisa Nacional por Amostra de Domicílios. [cited 2011 Oct 20]. Available from: http://www.bge.gov.br/home/estatistica/populacao/ trabalhoerendimento/pnad98/saude/analise.shtm

11. Castro DS. Estresse e estressores dos familiares de pacientes com traumatismo crânio encefálico em terapia intensiva [tese]. Rio de Janeiro (RJ): Escola de Enfermagem da Universidade Federal do Rio de Janeiro; 2009. 399 p.

12. Guirardello EB, Gabriel CAAR, Pereira IC, Miranda AF. A percepção do paciente sobre sua permanência na Unidade de Terapia Intensiva. Rev Esc Enferm USP. 1999;33(2):123-9.

13. Stiller K. Physiotherapy in intensive care: towards an evidence-based practice. Rev Chest. 2000;118(6):1801-13.

14. Netto BAB. Ministério da Saúde. [cited 2011 Jul O4]. Available from: http://dtr2001.saude.gov.br/sas/PORTARIAS/PORT98/GM/PRT-3432.pdf

15. Brasil. Ministério da Saúde [Internet]. Entendendo o SUS. [cited 2011 Dec 09]. Available from: http://portalsaude.saude.gov.br/portalsaude/ index.cfm/?portal=pagina.visualizarArea\&codArea=345

16. Brasil. Ministério da Saúde. Anvisa [Internet]. Resolução-RDC No 7 , de 24 de fevereiro de 2010. Diário Oficial da União, n 37, DOU de 25/02/2010 - seção 1 - p. 48. [cited 2012 Dec 12]. Available from: http://www.saude.mg.gov.br/atos_normativos/legislacao-sanitaria/ estabelecimentos-de-saude/uti/RDC-7_ANVISA\%20240210.pdf

17. Maruiti MR, Galdeano LE. Necessidades de familiares de pacientes internados em unidade de cuidados intensivos. Acta Paul Enferm. 2007:30(1):37-43.

18. Espinha TG, Amatuzzi MM. O cuidado e as vivências de internação em um hospital geral. Rev Psicol Teor Pesq. 2008:24(4):477-85.

19. Maciel MR, Souza MF. Acompanhante de adulto na unidade de terapia intensiva: uma visão do paciente. Rev Acta Paul Enferm. 2006:19(2):138-43

20. Urizzi F, Carvalho LM, Zamba HB, Ferreira GL, Grion CMC Cardoso LTQ. Vivência de familiares de pacientes internados em unidades de terapia intensiva. Rev Bras Ter Intensiva. 2008;20(4):370-5. 